\title{
64-MDCT Scan Demonstrated Pulmonary Vein Thrombus
}

\section{Hidekazu Takeuchi*}

Department of Cardiovascular Medicine, Yurinkai Higashi-Osaka Hospital, Osaka, Japan

\begin{abstract}
Although the relationship between deep venous thrombosis and pulmonary embolism is well known and studied extensively, the relationship between pulmonary vein thrombus (PVT) and systemic embolism such as cerebral infarction is unknown. 64-slice multidetector computed tomography (64-MDCT) scan can demonstrate PVT. We had reported two cases of small thrombus within the right upper pulmonary vein and the left upper pulmonary vein. A 74-year-old male with chronic atrial fibrillation was referred to be examined cardiac thrombus. 64-MDCT scan demonstrated large thrombi, which expanded from the right upper pulmonary vein to the left upper pulmonary vein and the left atrium. Three months warfarin therapy decreased the size of thrombi and changed the shape of thrombi. PVT is potentially life threatening condition and is thought to be a rare, but PVT seems to have been significantly underestimated. The 64-MDCT scan can assess PVT effectively and completely.
\end{abstract}

\section{Introduction}

Stroke is an important clinical problem of serious long-term disability and death, which is associated with significant morbidity, mortality, and socioeconomic impact. $20-25 \%$ of ischemic stroke is caused by immobilization of cardiogenic thrombi [1]. Atrial fibrillation (AF) is a strong independent risk factor against ischemic stroke [2]. The worst consequences of $\mathrm{AF}$ is ischemic stroke by anabolic thrombus, which is usually derived from the left atrium (LA) thrombus or left atrial appendage (LAA) thrombus [3,4].

Advancing age makes the prevalence of AF rise, from $0.1 \%$ in the patients less than age 55 to $9.0 \%$ in the patients more than age 80 . The patients with AF have a 5-fold increased risk of stroke compared with those without AF. Long-term anticoagulation lowers the risk of stroke by $64 \%$ overall in the patients with $\mathrm{AF}$ [5].

Thrombus of the patients with AF are mostly found during LAA and evaluation of LAA thrombus is commonly examined by transoesophageal echocardiography (TEE), which is a compromised tool and can't depict the 3D structure of the whole LAA, so TEE can't assess LAA thrombus completely. It is not easy to predict thromboembolic stroke according to the presence or absence of certain clinical and echocardiographic findings and plasma markers [6]. There is a possibility that the examination by using TEE is not sufficiently accurate to estimate the cardiac thrombus appropriately because TEE can't demonstrate the whole 3D reconstruction of LAA and pulmonary vein thrombus because of pulmonary air.

64-slice multidetector computed tomography (64-MDCT) scan is a valuable noninvasive tool to evaluate cardiac anatomy and function. 64-MDCT angiography has recently become an optional diagnostic method to estimate coronary artery plaque [7]. The 64-MDCT scan can also visualize the whole $3 \mathrm{D}$ reconstruction of the LAA in detail, and can assess the existence of thrombus in LAA completely [8].

The symptoms of PVT can be non-specific or subtle before occurring systemic thrombosis such as ischemic stroke, so the disease is often undiagnosed. The ways to detect the disease are searching. Pulmonary vein thrombus (PVT) has been reported after lung surgery such as pulmonary resection, lung transplantation or lobectomy [9], and radiofrequency catheter ablation for AF [10]. Although deep venous thrombosis (DVT) such as thrombus in popliteal vein, femoral vein and the inferior vena cave is well known as the cause of pulmonary (artery) embolism (PE), the relationship between PVT and systemic embolism such as cerebral infarction is unknown and PVT in the patients without lung surgery or catheter ablation is thought to be a rare. In 2012, for the first time, we reported two cases of small thrombus in the right upper pulmonary vein [11] or in the left upper pulmonary vein [12] in the patients without lung surgery or catheter ablation by using 64-MDCT. However, large thrombus of the pulmonary vein has not been reported.

We have identified large thrombi, which expand from the right upper pulmonary vein to the left upper pulmonary vein and left atrium (LA) in one AF patient without lung surgery or catheter ablation by 64-MDCT scan.

\section{Case Report}

The patient was a 74 year-old-male with chronic AF, and no previous treatment with warfarin had been performed. The patient was referred to our hospital for the evaluation of LAA thrombus. The patient had no symptoms of lung cancer or cerebral infarction. The chest roentgenogram showed no lung cancer. Large thrombi in the right upper pulmonary vein, the left upper pulmonary vein and LA were detected in axial (Figure 1a) and sagittal (Figure 2a) images as the defect of an enhancement by 64 -MDCT scan. The merge of thrombus is sharp (Figures $1 \mathrm{~b}$ and $2 \mathrm{c}$ ) and a part of thrombus could be seen in LA (Figures 1c, 2b).

After three months warfarin therapy, the size of the thrombi was decreased and the shape of the thrombi was changed (Figure $3 \mathrm{a}$ and Figure 4a). In Figure $3 \mathrm{~b}$ and Figure 4c, the sharp borderline was disappearing and the borderline became rather vague. In Figure $3 \mathrm{c}$, Figure $4 \mathrm{~b}$ and Figure $4 \mathrm{c}$, the size of thrombi in LA seemed to be decreased, which was depicted like "lines". During the warfarin therapy, the patient had no symptoms of systemic thrombosis such as cerebral infarction at all.

*Corresponding author: Hidekazu Takeuchi, Department of Cardiovascular Medicine, Yurinkai Higashi-Osaka Hospital, Osaka-city, Osaka 536-0005, Japan, Tel: +81-6-6939-1121; Fax: +81-6-6939-1191; E-mail: takeuchi-h@ymdmail.jp

Received January 18, 2013; Accepted February 14, 2013; Published February 16,2013

Citation: Takeuchi H (2012) 64-MDCT Scan Demonstrated Pulmonary Vein Thrombus. J Neurol Neurophysiol S8: 002. doi:10.4172/2155-9562.S8-002

Copyright: (c) 2012 Takeuchi H. This is an open-access article distributed under the terms of the Creative Commons Attribution License, which permits unrestricted use, distribution, and reproduction in any medium, provided the original author and source are credited. 
Citation: Takeuchi H (2012) 64-MDCT Scan Demonstrated Pulmonary Vein Thrombus. J Neurol Neurophysiol S8: 002. doi:10.4172/2155-9562.S8002
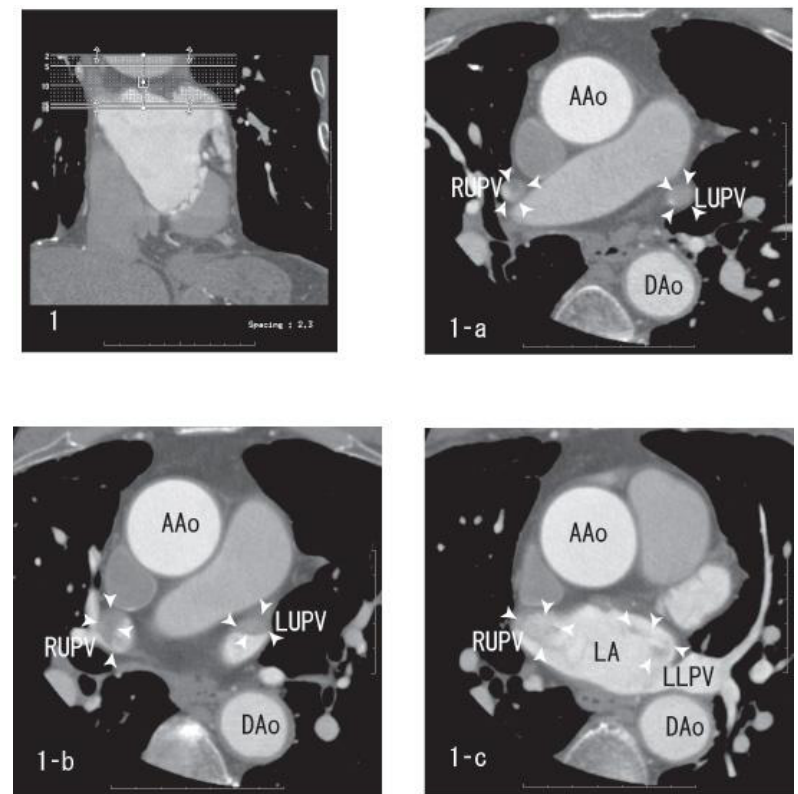

Figure 1: Axial images showing thrombi within the right upper and the left upper pulmonary veins (white arrow head). The right upper and the left upper pulmonary veins defected most of enhancement because of full sized thrombi (1-a). The left upper pulmonary vein demonstrated the sharp merge of enhancement (1-b). The merge of the thrombi in left atrium was vague (1-c). AAo: Ascending Aorta; DAo: Descending Aorta; LA: Left Atrium; LUPV: Left Upper Pulmonary Vein; LLPV: Left Lower Pulmonary Vein; RUPV: Right Upper Pulmonary Vein.
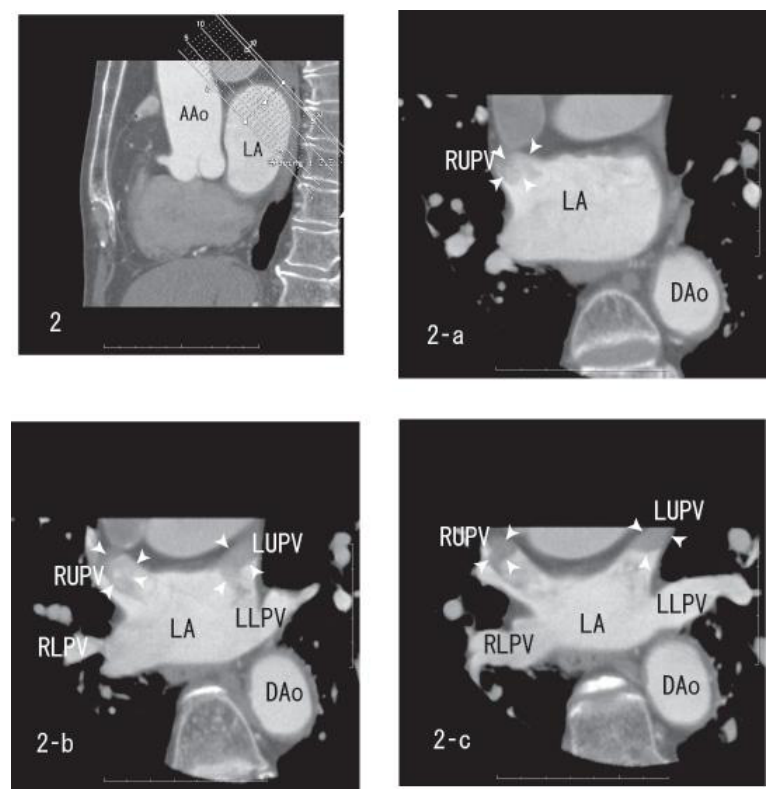

Figure 2: Sagittal images showing thrombi within the right upper and the left upper pulmonary veins (white arrow head). The merge of thrombi was vague (2-a and 2-b). The right upper and the left upper pulmonary veins demonstrated the sharp merge of enhancement (2-c). AAo: Ascending Aorta; DAo: Descending Aorta; LA: Left Atrium; LUPV: Left Upper Pulmonary Vein; LLPV: Left Lower Pulmonary Vein; RUPV: Right Upper Pulmonary Vein; RLPV: Right Lower Pulmonary Vein.

\section{Discussion}

In the present images, the right upper and the left upper pulmonary veins had the defect of enhancement, which were demonstrated in a dark color, and the borderline between white part and dark part was sharp before warfarin treatment, which suggested there was no blood flow in the right upper and the left upper pulmonary veins because large full sized thrombi in the right upper and the left upper pulmonary veins stop blood flow. What could stop blood flow of the right upper and the left upper pulmonary veins are mass such as thrombus or cancer. If there are no mass like thrombus or cancer in the right upper and the left upper pulmonary veins, the right upper and the left upper pulmonary veins should be demonstrated white like the right lower and the left lower pulmonary veins. And the patient had no lung cancer proof at all. So, the present images suggested that thrombi existed in both the right upper pulmonary vein and in the left upper pulmonary vein and in LA.

In the present case, we performed 64-MDCT scan again to assess the effects of three months warfarin therapy. The images after warfarin treatment demonstrated that the size of the thrombi was decreased and the shape of the thrombi was changed, but the most parts of fullsized thrombi were the similar shape, compared with the former shape. That's why full size thrombi stop the blood flow and warfare can't reach the most parts of thrombi, so there is no effect of warfarin therapy in the most parts of full-sized thrombi.

PVT has been reported after lung surgery [9], and radiofrequency catheter ablation for atrial fibrillation [10]. Certain tumor of the lung is one of the main causes of defect of enhancement and the extension itself of the tumor into proximal pulmonary veins, which make subsequently blood flow slow and thrombosis of the larger distal vein may look like PVT. And the present patient had no proof of lung cancer in the chest roentgenogram, in the 64-MDCT's images of both sides of middle to lower lung and in clinical symptoms.
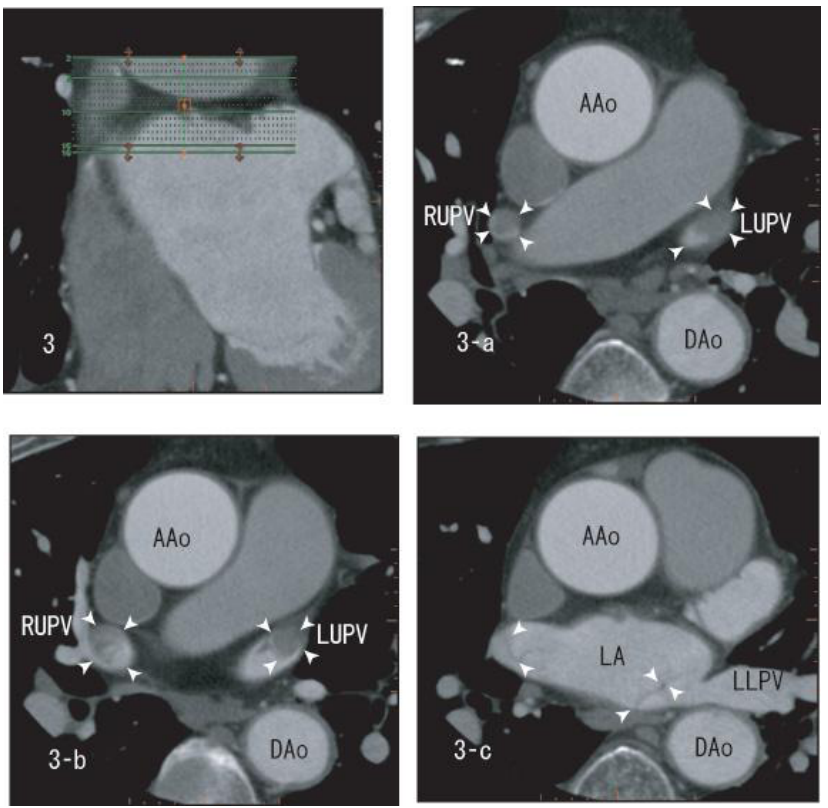

Figure 3: After three months warfarin therapy, axial images showing the thrombi within the right upper and the left upper pulmonary veins (white arrow head). The right upper and the left upper pulmonary veins defected the most of enhancement because of full sized thrombi (3-a). The sharp borderline of the left upper pulmonary vein was disappeared and the borderline became rather vague (3-b). The size of thrombi in LA seemed to be decreased and they looked like "lines" (3-c). AAo: Ascending Aorta; DAo: Descending Aorta; LA: Left Atrium; LUPV: Left Upper Pulmonary Vein; LLPV: Left Lower Pulmonary Vein; RUPV: Right Upper Pulmonary Vein. 

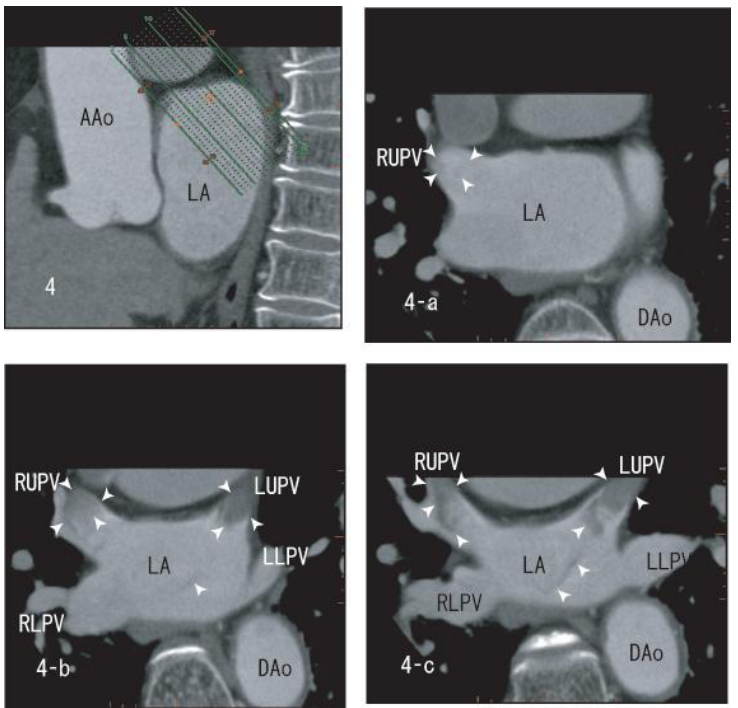

Figure 4: After three months warfarin therapy, sagittal images showing thromb within the right upper and the left upper pulmonary veins (white arrow head). The size of thrombi was decreased (4-a). Thrombi in the right upper and the left upper pulmonary veins demonstrated the change of the shape, and the size of thrombi in LA seemed to be decreased and they looked like "lines" (4-b and 4-c). AAo: Ascending Aorta; DAo: Descending Aorta; LA: Left Atrium; LUPV: Left Upper Pulmonary Vein; LLPV: Left Lower Pulmonary Vein; RUPV: Right Upper Pulmonary Vein; RLPV: Right Lower Pulmonary Vein.

For over 60 years, warfarin has been the main medication to prevent thromboembolic events in patients with AF. Although warfarin has clinical efficacy, warfarin has many limitations such as lots of interaction with other medications and food, so we need adjust dose by regular blood monitoring. More recently, we have other options such as rivaroxaban [13], dabigatran [14] and apixaban [15]. In the present case, warfarin had some effects on decreasing the pulmonary veins thrombi, but other new medications might have had a much more beneficial effect.

The images of LAA thrombus or pulmonary vein thrombus, which were demonstrated by 64 -MDCT scan, played an important role as a motivation to seek the medication for the patients with AF. And more 64-MDCT scan may be a useful tool to assess the effects of these medications.

As far as we know, this is the first to demonstrate large thrombi in the right upper and the left upper pulmonary veins in the patient without lung cancer or lung surgery, which could be detected by 64MDCT scan. 64-MDCT scan can be used to examine thrombus in not only LAA but the pulmonary vein.

The 64-MDCT scan may play an important role in estimating the effects of anticoagulants such as warfarin because 64-MDCT scan can demonstrate PVT completely in spite of pulmonary air.

Despite the large thrombi in the right upper and the left upper pulmonary veins, the patient had no symptom of systemic thrombosis such as cerebral infarction at all. The character of thrombus in pulmonary vein, LA or LAA may be different from thrombus forming DVT. A part of thrombus of DVT is often separated from DVT and goes into the pulmonary artery, which causes PE. But thrombus of PVT seems not to be separated so easily. There is a possibility that cardiac thrombi don't cause stroke so occasionally.

PVT is potentially life threatening condition and is thought to be a rare. But we have experienced another small thrombus in the pulmonary vein of the patients without lung surgery or lung cancer $[11,12]$, so PVT may not be a rare. More attention should be paid to PVT and much more studies about PVT are desirable to avert ischemic stroke.

The examination by 64-MDCT scan could help the doctors to recognize the existence of pulmonary vein thrombus and to prevent the patients from systemic thrombosis such as cerebral infarction.

\section{Conclusion}

As far as we know, this is the first to demonstrate large full sized thrombi, which extended from the right upper pulmonary vein to the left upper pulmonary vein and LA for the patient without lung surgery or lung cancer detected by 64-MDCT scan. More attention should be paid to PVT for the prevention of cardioembolic stroke because PVT may not be a rare.

64-MDCT scan may play an important role in estimating the effects of anticoagulants because 64-MDCT scan can demonstrate PVT completely in spite of pulmonary air. Despite the large thrombi in the right and the left pulmonary veins, the patient had no systemic thrombosis such as cerebral infarction.

\section{References}

1. Olgin JE, Zipes DP (2005) Specific arrhythmias: diagnosis and treatment. In Zipes DP, Libby P, Bonow RO, Braunwald E (eds.), Braunwald's heart disease. (7thedn.) Pennsylvania: Elsevier Saunders Philadelphia, USA.

2. Ismail D (2004) Peran antikoagulan pada FA. Pendekatan holistik penyakit kardiovaskular IV. Jakarta: Pusat Penerbitan IImu Penyakit Dalam FKUI; $97-$ 102.

3. Page RL (2004) Clinical practice. Newly diagnosed atrial fibrillation. N Engl Med 351: 2408-2416

4. Benjamin EJ, D’Agostino RB, Belanger AJ, Wolf PA, Levy D (1995) Left atria size and the risk of stroke and death. The Framingham Heart Study. Circulation 92: 835-841.

5. Hart RG, Pearce LA, Aguilar MI (2007) Meta-analysis: antithrombotic therapy to prevent stroke in patients who have nonvalvular atrial fibrillation. Ann Intern Med 146: 857-867.

6. Goldman ME, Pearce LA, Hart RG, Zabalgoitia M, Asinger RW, et al. (1999) Pathophysiologic correlates of thromboembolism in nonvalvular atrial fibrillation: I. Reduced flow velocity in the left atrial appendage (The Stroke Prevention in Atrial Fibrillation [SPAF-III] study). J Am Soc Echocardiogr 12: 1080-1087.

7. Takeuchi H (2012) Warfarin improved CT values of the coronary artery artherosclerotic plaque: evaluation by 64-MDCTA. BMJ Case Rep Sep 24;2012. doi:pii: bcr0220125941. 10.1136/bcr.02.2012.5941.

8. Takeuchi H (2011) A 64-slice multi-detector CT scan could evaluate the change of the left atrial appendage thrombi of the atrial fibrillation patient, which was reduced by warfarin therapy BMJ Case Rep Aug 19;2011. doi:pii: bcr1120092491. 10.1136/bcr.11.2009.2491.

9. Genta PR, Ho N, Beyruti R, Takagaki TY, Terra-Filho M (2003) Pulmonary vein thrombosis after bilobectomy and development of collateral circulation. Thorax 58: 550-551.

10. Yataco J, Stoller JK (2004) Pulmonary venous thrombosis and infarction complicating pulmonary venous stenosis following radiofrequency ablation. Respir Care 49: 1525-1527.

11. Takeuchi $H$ (2012) A thrombus of the right upper pulmonary vein: detection by the use of a 64-MDCT. BMJ Case Rep 2012. doi:pii: bcr1220115446. 10.1136/ bcr.12.2011.5446

12. Takeuchi H (2012) 64-MDCT showed the thrombus in the pulmonary vein of the patient with angina pectoris BMJ Case Rep Oct 13;2012. doi:pii: bcr0220125839. 10.1136/bcr.02.2012.5839.

13. Patel MR, Mahaffey KW, Garg J, Pan G, Singer DE, et al. (2011) Rivaroxaban versus warfarin in nonvalvular atrial fibrillation. N Engl J Med 365: 883-891. 
Citation: Takeuchi H (2012) 64-MDCT Scan Demonstrated Pulmonary Vein Thrombus. J Neurol Neurophysiol S8: 002. doi:10.4172/2155-9562.S8002

14. Connolly SJ, Ezekowitz MD, Yusuf S, Eikelboom J, Oldgren J, et al. (2009) Dabigatran versus warfarin in patients with atrial fibrillation. N Engl J Med 361: 1139-1151.
15. Granger CB, Alexander JH, McMurray JJ, Lopes RD, Hylek EM, et al. (2011) Apixaban versus warfarin in patients with atrial fibrillation. $\mathrm{N}$ Engl J Med 365: 981-992.

This article was originally published in a special issue, Stroke:

Cerebrovascular accident handled by Editor(s). Dr. David Della Morte,

University of Miami, USA 